\title{
Ensino de práticas integrativas e complementares: uma análise dos cursos de
} Odontologia do Brasil

\author{
Teaching integrative and complementary practices: an analysis of Dentistry courses in Brazil \\ Enseñanza de prácticas integrativas y complementarias: un análisis de cursos de Odontología en \\ Brasil
}

Jessika Paiva Medeiros ORCID: https://orcid.org/0000-0001-6624-812X Universidade Federal de Campina Grande, Brasil E-mail: jessikapaiva21@gmail.com

Júlia Kiara da Nóbrega Holanda ORCID: https://orcid.org/0000-0002-1426-0714 Universidade Federal de Campina Grande, Brasil E-mail: juliakiaranh@gmail.com

Josilanny Araújo de Souza Alencar ORCID: https://orcid.org/0000-0001-6539-0773 Universidade Federal de Campina Grande, Brasil E-mail: joylany@hotmail.com

Ana Caroline Rodrigues ORCID: https://orcid.org/0000-0002-1945-9132 Universidade Federal de Campina Grande, Brasil

E-mail: rodriguesanacaroline252@gmail.com

Aléxia Araújo Alencar

ORCID: https://orcid.org/0000-0002-3030-9901

Universidade Federal de Campina Grande, Brasil E-mail: alexia.alencarr@hotmail.com

Lara Danúbia Galvão de Souza ORCID: https://orcid.org/0000-0001-5693-9453 Universidade Federal de Campina Grande, Brasil E-mail: lara.danubia@outlook.com Lívia da Silva Pereira

ORCID: https://orcid.org/0000-0003-2057-3570 Universidade Federal de Campina Grande, Brasil E-mail: liviadasilva9990@gmail.com

Ana Beatriz Bomfim Gomes Ribeiro ORCID: https://orcid.org/0000-0001-9069-2386 Universidade Federal de Campina Grande, Brasil E-mail: abeatrizbomfim98@gmail.com

Viton Dyrk Guimarães Fernandes ORCID: https://orcid.org/0000-0003-4901-7391 Universidade Federal de Campina Grande, Brasil E-mail: vitondyrk@gmail.com

Maria Angélica Sátyro Gomes Alves ORCID: https://orcid.org/0000-0003-3329-8360 Universidade Federal de Campina Grande, Brasil E-mail: angelicasatyro@hotmail.com

Gymenna Maria Tenório Guênes ORCID: https://orcid.org/0000-0002-5447-0193 Universidade Federal de Campina Grande, Brasil E-mail: gymennat@yahoo.com.br

Luanna Abílio Diniz Melquíades de Medeiros ORCID: https://orcid.org/0000-0002-1630-3968 Universidade Federal de Campina Grande, Brasil

E-mail: luannaabiliod@gmail.com

Elizandra Silva da Penha ORCID: https://orcid.org/0000-0001-6264-5232 Universidade Federal de Campina Grande, Brasil E-mail: elizandrapenha@ hotmail.com 


\author{
Raline Mendonça dos Anjos \\ ORCID: https://orcid.org/0000-0003-0751-7523 \\ Universidade Federal de Campina Grande, Brasil \\ E-mail: raline.anjos@gmail.com \\ Abrahão Alves de Oliveira Filho \\ ORCID: https://orcid.org/0000-0002-7466-9933 \\ Universidade Federal de Campina Grande, Brasil \\ E-mail: abrahao.farm@gmail.com
}

\begin{abstract}
Resumo
Este estudo tem como objetivo avaliar a oferta de disciplinas referente ao ensino das práticas integrativas e complementares (PICS) nos cursos de Odontologia do Brasil. Efetuou-se uma pesquisa de caráter transversal, quantitativo e exploratório, por meio dos dados fornecidos pelo Cadastro e-MEC, do Ministério da Educação, no período de janeiro a março de 2021. Foram consultadas todas as Instituições de Ensino Superior em situação ativa, com endereço eletrônico oficial, matriz curricular disponível ou Projeto Pedagógico do Curso. Após aplicação dos critérios de inclusão e exclusão, 228 cursos foram selecionados para verificar a categoria administrativa da universidade, a localização do curso e a inserção de disciplinas referentes ao ensino das PICS que, quando identificado, foi avaliado quanto a sua natureza e carga horária total. Os dados coletados foram tabulados e tratados de forma descritiva. Considerando os cursos com matriz curricular completa disponível, apenas $10(4,39 \%)$ ofertaram disciplinas referentes ao ensino das PICS, enquanto a maioria da amostra se encontrava ausente na grade curricular $(218 ; 95,61 \%)$. As regiões Norte, Centro-Oeste $(1 ; 10 \%)$, Nordeste $(2 ; 20 \%)$, Sudeste $(6 ; 60 \%)$ e Sul $(1 ; 10 \%)$ apresentaram cursos ofertando a disciplina, de natureza obrigatória em apenas uma matriz curricular e com cargas horárias variando entre $30 \mathrm{~h}$ e $60 \mathrm{~h}$. Fica evidente que o ensino das PICS ainda é escasso no Brasil, afetando indiretamente o Sistema Público de Saúde, uma vez que o perfil de egresso do cirurgião-dentista ainda é centrado no modelo biomédico, dificultando as políticas e o incentivo do uso de alternativas complementares no SUS.
\end{abstract}

Palavras-chave: Odontologia; Práticas integrativas e complementares; Terapêutica.

\begin{abstract}
This study aims to evaluate the offer of disciplines related to the teaching of integrative and complementary practices (PICS) in Dentistry courses in Brazil. A cross-sectional, quantitative and exploratory research was carried out, using data provided by the e-MEC Registry, of the Ministry of Education, from January to March 2021. All Higher Education Institutions in active situation were consulted, with official e-mail address, available curriculum matrix or Pedagogical Project of the Course. After applying the inclusion and exclusion criteria, 228 courses were selected to verify the administrative category of the university, the location of the course and the inclusion of subjects related to the teaching of PICS which, when identified, were evaluated for their nature and total workload. The collected data were tabulated and treated descriptively. Considering courses with a complete curriculum available, only $10(4.39 \%)$ offered subjects related to teaching PICS, while the majority of the sample was absent from the curriculum (218; $95.61 \%)$. The North, Central-West $(1 ; 10 \%)$, Northeast $(2 ; 20 \%)$, Southeast $(6 ; 60 \%)$ and South $(1 ; 10 \%)$ regions had courses offering the subject, of a mandatory nature in only one matrix curricular and with hourly loads varying between 30h and 60h. It is evident that the teaching of PICS is still scarce in Brazil, indirectly affecting the Public Health System, since the egress profile of dental surgeons is still centered on the biomedical model, hindering policies and encouraging the use of complementary alternatives in the SUS.
\end{abstract}

Keywords: Dentistry; Integrative and complementary practices; Therapeutics.

\title{
Resumen
}

Este estudio tiene como objetivo evaluar la oferta de disciplinas relacionadas con la enseñanza de prácticas integradoras y complementarias (PICS) en los cursos de Odontología en Brasil. Se realizó una investigación transversal, cuantitativa y exploratoria, utilizando datos proporcionados por el Registro e-MEC, del Ministerio de Educación, de enero a marzo de 2021. Se consultó a todas las Instituciones de Educación Superior en situación activa, con correo electrónico oficial. dirección, matriz curricular disponible o Proyecto Pedagógico del Curso. Luego de aplicar los criterios de inclusión y exclusión, se seleccionaron 228 cursos para verificar la categoría administrativa de la universidad, la ubicación del curso y la inclusión de asignaturas relacionadas con la docencia de PICS que, al ser identificadas, fueron evaluadas por su naturaleza y carga total de trabajo. . Los datos recopilados se tabularon y trataron de forma descriptiva. Considerando cursos con un plan de estudios completo disponible, solo $10(4,39 \%)$ ofrecieron asignaturas relacionadas con la enseñanza de PICS, mientras que la mayoría de la muestra estuvo ausente del plan de estudios $(218 ; 95,61 \%)$. Las regiones Norte, Centro-Oeste $(1 ; 10 \%)$, Noreste $(2 ; 20 \%)$, Sudeste $(6 ; 60 \%)$ y Sur $(1 ; 10 \%)$ contaban con cursos que ofrecían la asignatura, de carácter obligatorio en solo una matriz curricular y con cargas horarias que varían entre 30h y $60 \mathrm{~h}$. Es evidente que la enseñanza del PICS aún es escasa en Brasil, afectando indirectamente al Sistema Público de Salud, ya que el perfil de egreso de los dentistas todavía se centra en el modelo biomédico, dificultando las políticas y fomentando el uso de alternativas complementarias en el SUS.

Palabras clave: Odontología; Prácticas integrativas y complementarias; Terapéutica. 


\section{Introdução}

As Práticas Integrativas e Complementares consistem em uma atividade terapêutica baseada em teorias voltadas para os aspectos ambientais e comportamentais do processo saúde-doença (Habimorad et al., 2020). Fundamentada na necessidade de garantir a segurança dos pacientes, qualidade e eficácia, a discussão sobre a formação para o exercício profíssional nesta área tem ganhado relevância (Sousa et al., 2012).

Em 2006, o Ministério da Saúde aprovou a Política Nacional de Práticas Integrativas e Complementares no SUS (PNPIC) com a justificativa embasada na garantia do princípio da integralidade. A principal finalidade é atender a necessidade de conhecer, apoiar e incorporar as experiências com PICS já em andamento na rede pública de saúde, visando estimular os mecanismos naturais de prevenção, recuperação e promoção da saúde. No entanto, ainda existe um baixo conhecimento por parte dos profissionais e gestores do SUS em relação à PNPIC, o que constitui uma fragilidade da política (Habimorad et al., 2020).

$\mathrm{Na}$ odontologia, parte dos pacientes faz uso de terapias alternativas e complementares, com maior destaque para a fitoterapia e técnicas mente-corpo em casos de indivíduos que lidam com ansiedade quando submetidos a um tratamento odontológico (Spector et al., 2012). Cirurgiões-dentistas alemães têm se destacado por fazer uso em sua rotina clínica da fitoterapia a partir da utilização da Arnica Montana (64\%), camomila (64\%), cravo (63\%), Salvia officinalis (54\%), seguida das abordagens de relaxamento (62\%), bem como da homeopatia (57\%). Destarte, é evidente que ainda há uma lacuna entre a falta de evidências científicas para a maioria das práticas integrativas complementares em odontologia e a prática clínica (Baatsch et al., 2017).

Nesse contexto, a falta de disciplinas centradas nas PICS durante a graduação do estudante de odontologia, permite construir um perfil acadêmico e profissional com competências, habilidades e conteúdo dentro de perspectivas e abordagens contemporâneas alicerçadas no modelo biomédico (Brasil, 2001). Dessa forma, cabe ao profissional diante da necessidade, aprofundar-se mais sobre o tema, recorrendo a cursos de pós-graduação, cursos de extensão ou até mesmo aperfeiçoamento (Santos, 2018).

Em vista do exposto, este trabalho tem como objetivo fazer uma análise do ensino das terapias alternativas e complementares em relação à presença de tal disciplina nas matrizes curriculares dos cursos de odontologia do Brasil.

\section{Metodologia}

Esta pesquisa consiste em um estudo de caráter transversal, quantitativo e descritivo, realizado no período de janeiro a março de 2021, a partir de dados obtidos no Cadastro Nacional de Cursos e Instituições de Educação Superior (Cadastro eMEC) no site eletrônico do Ministério da Educação (Brasil, 2021). Dessa forma, foram consultadas as Instituições de Ensino Superior (IES) em situação ativa, públicas e privadas, em todas as regiões brasileiras.

Após consulta no e-MEC, foi realizada a busca pelos sítios eletrônicos das universidades, com o objetivo de verificar a presença de componentes curriculares relativos ao ensino das Práticas Integrativas e Complementares em Saúde (PICS). Foram excluídos da amostra os cursos que não possuíam um endereço eletrônico oficial, os que não disponibilizaram a grade curricular ou que, quando disponível, não especificaram quais as disciplinas optativas ofertadas. Para inclusão no estudo, foram considerados os cursos em que foi possível observar a grade curricular completa.

Assim, o objeto de estudo compreendeu os 556 cursos de Odontologia das IES do Brasil, ativos no momento da pesquisa. Contudo, após a aplicação dos critérios de inclusão e exclusão referidos, obteve-se uma amostra de 228 cursos. Posteriormente, os pesquisadores verificaram a categoria administrativa da universidade (pública ou privada), a localização do 
curso e a inserção de disciplinas referentes ao ensino das PICS. Quando presente, o componente curricular foi avaliado quanto à sua natureza (obrigatória ou optativa) e a carga horária total.

Os dados coletados foram tabulados no programa de informática Statistical Package for Social Sciences (SPSS) para Windows, versão 13.0, e tratados de forma descritiva.

\section{Resultados}

Participaram deste estudo 556 instituições de ensino superior, sendo considerados os cursos de diferentes polos de uma mesma IES. Dentre elas, 142 (25,54\%) não forneceram acesso a matriz curricular do curso de Odontologia em endereço eletrônico; enquanto 414 (74,46\%) instituições deferiram acesso, das quais apenas 228 (55,07\%) apresentaram a matriz curricular completa, contendo disciplinas obrigatórias e optativas especificadas, compondo a amostra final do estudo, o que representou $41 \%$ dos cursos de Odontologia ativos no Brasil.

Dessa forma, de todos os cursos que apresentaram matriz curricular completa em seus sítios eletrônicos, apenas 10 $(4,39 \%)$ ofertaram disciplinas referentes ao ensino das PICS, enquanto a maioria $(218 ; 95,61 \%)$ não ofertou (Figura 1$)$.

Figura 1. Instituições de Ensino Superior do Brasil.



Fonte: Autores (2021).

Quando observado por região (Tabela 1), a oferta das práticas integrativas e complementares na graduação de cirurgiões-dentistas esteve presente no Centro-Oeste $(1 ; 10 \%)$, Nordeste $(2 ; 20 \%)$, Sudeste $(6 ; 60 \%)$ e Sul (1; 10\%), estando ausente no Norte do país. 
Tabela 1. Total de cursos públicas e privados do Brasil que ofertam disciplinas de Práticas Integrativas e Complementares em 2021.

\begin{tabular}{lc}
\hline Região & Oferta de PICS \\
\hline Centro-Oeste & $\mathbf{1}$ \\
Nordeste & 2 \\
Norte & $\mathbf{0}$ \\
Sudeste & 6 \\
Sul & 1 \\
Total & 10 \\
\hline
\end{tabular}

Fonte: Autores (2021).

No que se refere à categoria administrativa, apenas $3(30 \%)$ cursos são de IES públicas, enquanto a maioria (7; 70\%) são de IES privadas. A carga horária variou de 30 a 60 horas-aula, sendo a disciplina de natureza obrigatória em apenas uma matriz curricular (Tabela 2).

Tabela 2. Divisão das faculdades que possuem a disciplina em (Pública/Privada) (Optativa/Obrigatória) e suas respectivas cargas horárias- 2021.

\begin{tabular}{|c|c|c|c|}
\hline $\begin{array}{c}\text { Instituição de } \\
\text { Ensino Superior }\end{array}$ & $\begin{array}{c}\text { Categoria } \\
\text { administrativa }\end{array}$ & $\begin{array}{c}\text { Natureza } \\
\text { da disciplina }\end{array}$ & Carga Horária \\
\hline UFRJ & Público & Optativa & $45 \mathrm{~h}$ \\
\hline UFPR & Público & Optativa & $60 \mathrm{~h}$ \\
\hline PUC-Goiás & Privado & Optativa & $30 \mathrm{~h}$ \\
\hline Multivix-Vila Velha & Privado & Optativa & Não informou \\
\hline Multivix-Cachoeiro & Privado & Optativa & Não informou \\
\hline Multivix-Nova Venécia & Privado & Optativa & Não informou \\
\hline Multivix-Vitória & Privado & Optativa & Não informou \\
\hline UEPB-Campina Grande & Público & Optativa & $30 \mathrm{~h}$ \\
\hline Faculdade Santa Maria & Privado & Obrigatória & $45 \mathrm{~h}$ \\
\hline
\end{tabular}

Fonte: Autores (2021).

\section{Discussão}

Estas Práticas Integrativas e complementares apresentam ênfase em sistemas e recursos que envolvem abordagens com o intuito de buscar e estimular o desenvolvimento de mecanismos naturais de prevenção de agravos e recuperação da saúde, com tecnologias eficazes e seguras, além de expandir o vínculo terapêutico e a integração do ser humano com o meio ambiente e a sociedade, promovendo uma ampliação do processo saúde-doença. (BRASIL, 2015; GOLÇALVES et al., 2018).

A Resolução CNE/CES 3, de 19 de fevereiro de 2002, no artigo 3, afirma que o perfil do futuro cirurgião-dentista, com base no rigor técnico e científico da sua formação generalista, humanista, crítica e reflexiva, está apto para atuar em todos os níveis de atenção à saúde, estando, desse modo, capacitado ao exercício de atividades voltadas à saúde bucal da população, levando em consideração a realidade social, cultural e econômica do seu meio. Levando isso em consideração, percebe-se a importância de o formado egresso receber em sua formação, paradigmas que abordam as PICs já que, além de outros fatores, a 
literatura científica aponta uma melhor contribuição no relacionamento com os pacientes. Ademais, diminui abordagens invasivas e insensíveis, amplia a integralidade e torna o trabalho em saúde mais resolutivo (BRASIL, 2002; NASCIMENTO et al., 2018).

Após a análise das grades curriculares dos cursos de odontologia, percebeu-se que essa área de ensino foi ofertada, em sua maioria, como disciplina optativa nas universidades. Desde 1993, Schraiber afirma que a adoção de tais práticas enfrenta obstáculos tanto técnicos e científicos quanto ideológicos e econômicos já que a conformação das práticas hegemônicas e da formação dos profissionais de saúde sofre grande influência de um ideário que desqualifica as iniciativas que se afastam do modelo conhecido como medicina tecnológica. Logo, esse fator, atribuído à falta de conhecimento, interesse, confiança ou não formação específica durante a graduação, reflete a deficiência desse componente curricular nos cursos de odontologia em um país que possui políticas e incentiva o uso de alternativas complementares no sistema público de saúde (JUNIOR; MONTEIRO, 2020).

De acordo com Nascimento et al. (2018), ainda existe uma escassa oferta de informações organizadas sobre a oferta de ensino em PICs, no Brasil. Ademais, grande parte das áreas de estudo aborda o tema em poucas instituições locais e a ausência de formação de egressos neste tema é ampla, o que demonstra a necessidade de ampliação das pesquisas nessa temática. Atrelado a isso, a falta de ensino acaba prejudicando a prescrição segura para a tomada de decisões em relação ao seu uso, como indicações terapêuticas, mecanismos de ação, interações medicamentosas e possíveis riscos, gerando uma barreira entre o uso dessas práticas e o paciente.

Em seu estudo, Gontijo e Nunes (2017) constataram que dos 118 participantes (78,7\%), dentre eles, médicos, enfermeiros, fisioterapeutas, biomédicos, psicólogos, assistentes sociais, nutricionistas, farmacêuticos e cirurgiões-dentistas, 92,4\% consideram as PICs eficientes, 86,4\% afirmaram ser importantes para a profissão, 94,9\%, necessário para o SUS e apenas 30,5\% atribuíram suas considerações ao que foi ensinado durante a graduação, ratificando os dados encontrados neste estudo e as análises realizadas anteriormente.

À vista disso, fica claro a necessidade da expansão institucionalmente e socialmente das Práticas Integrativas e Complementares, com maior elucidação da conduta e importância no meio acadêmico, para possibilitar um foco maior para o tema, além da troca de experiência que serão incorporadas à rotina de trabalho como um processo efetivo, contínuo e ajustado às necessidades do Sistema Único de Saúde, valorizando a integralidade em saúde e a promoção global do cuidado humano (SAVARIS et al., 2019).

\section{Conclusão}

O ensino das práticas integrativas e complementares ainda é escasso nos cursos de odontologia do Brasil. Além disso, de acordo com o presente estudo, a disciplina quando ofertada nas IES, esteve presente em sua maioria em universidades privadas apresentando-se com natureza optativa. Dessa forma, indiretamente, o sistema público de saúde é afetado, uma vez que o perfil de egresso do cirurgião-dentista ainda é centrado no modelo biomédico, o que dificulta as políticas e o incentivo do uso de alternativas complementares no SUS. Sendo assim, é necessário expandir o uso das PICS nos cursos de graduação em odontologia, para que os futuros cirurgiões-dentistas se sintam seguros para possíveis indicações terapêuticas, bem como interações medicamentosas, além de facilitar a integração do ser humano com o meio ambiente e a sociedade. 
Research, Society and Development, v. 11, n. 1, e0511123264, 2022

(CC BY 4.0) | ISSN 2525-3409 | DOI: http://dx.doi.org/10.33448/rsd-v11i1.23264

\section{Referências}

Baatsch, B., Zimmer, S., Recchia, D. R., \& Büssing, A. (2017). Complementary and alternative therapies in dentistry and characteristics of dentists who recommend them. Complementary therapies in medicine, 35, 64-69.

BRASIL. Ministério Da Educação. Diretrizes Curriculares Nacionais dos Cursos de Graduação em Enfermagem, Medicina e Nutrição. Brasília, Conselho Nacional de Educação, 2001. Disponível em: http://portal.mec.gov.br/dmdocuments/ces1133.pdf.

BRASIL. Conselho Nacional de Educação. Resolução No CNE/CNS 3, de 19 de fevereiro de 2002. Institui Diretrizes Curriculares Nacionais do Curso de Graduação em Odontologia. Diário Oficial da União. Seção 1. Brasília, DF. 2002;(42):10-11.

BRASIL. Ministério da Educação. Cadastro e-MEC de Instituições e Cursos de Educação Superior. [acesso em: 12/01/2021]. Disponível em: http://emec.mec.gov.br/.

BRASIL. Ministério da Saúde (MS). Secretaria de Atenção à Saúde. Departamento de Atenção Básica. Política Nacional de Práticas Integrativas e Complementares no SUS - PNPIC-SUS. Brasília: MS; 2006 (Série B. Textos Básicos de Saúde).

BRASIL. Ministério da Saúde (MS). Secretaria de Ciência, Tecnologia e Insumos Estratégicos. Departamento de Assistência Farmacêutica. Política Nacional de Plantas Medicinais e Fitoterápicos. Brasília: MS; 2006 (Série B. Textos Básicos de Saúde).

BRASIL. Ministério da Saúde. Secretaria de Atenção à Saúde. Departamento de Atenção Básica. Política nacional de práticas integrativas e complementares no SUS: atitude de ampliação de acesso / Ministério da Saúde. Secretaria de Atenção à Saúde. Departamento de Atenção Básica. - 2. ed. - Brasília: Ministério da Saúde, 2015

Gonçalves, R. N., Gonçalves, J. R. D. S. N., Buffon, M. D. C. M., Negrelle, R. R. B., \& de Albuquerque, G. S. C. (2018). Práticas Integrativas e Complementares: inserção no contexto do ensino Odontológico. Revista da ABENO, 18(2), 114-123.

Gontijo, M. B. A., \& Nunes, M. D. F. (2017). Práticas integrativas e complementares: conhecimento e credibilidade de profissionais do serviço público de saúde. Trabalho, Educação e Saúde, 15, 301-320.

Habimorad, P. H. L., Catarucci, F. M., Bruno, V. H. T., Silva, I. B. D., Fernandes, V. C., Demarzo, M. M. P., Spagnuolo, R. S., \& Patricio, K. P. (2020). Potencialidades e fragilidades de implantação da Política Nacional de Práticas Integrativas e Complementares. Ciência \& Saúde Coletiva, $25,395-405$.

Junior, J. I., \& Monteiro, Á. B. (2020). Plantas medicinais e fitoterápicos úteis na odontologia clínica: uma revisão medicinal. Revista da Faculdade de Odontologia da UFBA, 50(1), 47-56.

Nascimento, M. C. D., Romano, V. F., Chazan, A. C. S., \& Quaresma, C. H. (2018). Formação em práticas integrativas e complementares em saúde: desafios para as universidades públicas. Trabalho, Educação e Saúde, 16(2), 751-772.

Santos, G.M., Nogueira, T.A., Monteiro, M.J.S.D., Barreto, M.T.S., Oliveira, J.F. (2018). A fitoterapia na formação do profissional nutricionista. Brazilian Journal of Surgery and Clinical Research, 25(1), 49-52.

Savaris, L. E., Boger, B., Savian, A. C., Jansen, A. S., Silva, M. Z. (2019). Práticas integrativas e complementares- análise documental e o olhar de profissionais da saúde. Rer. Bras. Promoç. Saúde.

Schraiber, L. B. (1993). O médico e seu trabalho: limites da liberdade. In O médico e seu trabalho: limites da liberdade (pp. 229-229).

Sousa, I. M. C. D., Bodstein, R. C. D. A., Tesser, C. D., Santos, F. D. A. D. S., \& Hortale, V. A. (2012). Práticas integrati vas e complementares: oferta e produção de atendimentos no SUS e em municípios selecionados. Cadernos de Saúde Pública, 28, 2143-2154.

Spector, M. L., Fischer, M., Dawson, D. V., Holmes, D. C., Kummet, C., Nisly, N. L., \& Baker, K. A. (2012). Complementary and alternative medicine usage by patients of a dental school clinic. Special Care in Dentistry, 32(5), 177-183. 